\title{
A New Controller Design Method for Single Loop Internal Model Control Systems
}

\author{
Arun RAMAVEERAPATHIRAN ${ }^{1}$, Muniraj RATHINAM ${ }^{2 *}$, \\ Willjuice Iruthayarajan MARIA SILUVAIRAJ ${ }^{2}$ \\ ${ }^{1}$ Department of Electrical and Electronics Technology, Federal TVET Institute, \\ Addis Ababa, Ethiopia \\ arun.pathiran@gmail.com \\ ${ }^{2}$ Department of Electrical and Electronics Engineering, National Engineering College, Kovilpatti, \\ Affiliated to Anna University, Chennai, Tamilnadu, India \\ munirajphd@gmail.com (*Corresponding author),m.willjuice@gmail.com
}

\begin{abstract}
In the present developed work, a new controller design methodology of Internal Model Controller has been proposed for improving the closed-loop response. The IMC controller has been viewed into its gain and dynamic parts. The key strategic difference is that the controller dynamic part has a generalized structure. The designer has the choice to select between low and high-order controllers to outfit the control applications better. The controller order has been considered in terms of simple first/second-order type, and the design guidelines are presented. As in the case of the traditional IMC controller, there is principally a single variable parameter in the developed IMC controller, which can be monotonically tuned to meet the compromise between achievable performance and the stability of a closed-loop system. The merits and applications of the developed IMC controller design methodology for high-order systems have been evaluated using several simulation examples.
\end{abstract}

Keywords: Internal Model Controller (IMC), First Order Plus Dead Time (FOPDT) Process Model, Internal Absolute Error (IAE), SIMC-PI Controller, SIMC-PID Controller.

\section{Introduction}

The IMC theory was presented in the 1980s by Morari and his associated researchers (Morari \& Zafiriou, 1989). The IMC system has several advantages compared to the conventional feedback control system (Arun \& Praksh, 2018). The IMC approach allows uncertainty in model and tradeoffs between performance/robustness to be considered in a more efficient fashion (Seborg et al., 2004). The approach of IMC was successfully employed and executed in a number of applications (Van de Vegte, 1986) such as powering control (Rupp \& Guzzella, 2010) synchronous motor speed regulation $(\mathrm{Li} \& \mathrm{Gu}$, 2012), load frequency control of power system (Saxena \& Hote, 2013), temperature control (Yadav \& Gaur, 2013), vehicle yaw control (Canale et al., 2009) and sun-seeker system (Jha et al., 2014).

The design of a standard IMC controller is reperesented by the controller defined as the inverse of the invertible part of the model (i.e., minimum phase elements) and further augmented by a low-pass filter employed to generate a practicable controller. The filter part of the IMC controller can be selected to meet the desired reference tracking response (Zitek \& Halva, 2001). A very popular selection of a filter with critical damping is $\frac{1}{(\lambda s+1)^{n}}$. The filter transfer

https://doi.org/10.24846/v29i2y202007 function order ' $n$ ', is chosen with respect to the order of the process transfer function. The appropriate selection of tuning parameter $\lambda$ can achieve either the desired performance/robustness (Shamsuzzoha \& Lee, 2007). Thus, the design of typical IMC controller can therefore generally be condensed to one parameter optimization.

However, the standard low pass filter shows an undesirable regulatory response for processes with minimum dead-time to time-constant ratio. This drawback is detailed in various papers (Wang \& Zhang 2001; Lennartson \& Kristiannson, 2009) and solutions for maximizing the performance of disturbance rejection have been discussed (Liu \& Gao 2010; Alcantara et al., 2010 ). Further, the conventional IMC technique relies on pole-zero cancellation that is rare in practice. In practical, it is very challenging to have a perfect pole-zero cancellation (Chau, 2002). The commonly selected filter and its modified structures may not be the optimal choice concerning the constraints in the particular type of performance (Lennartson \& Kristiannson, 2009). Hence, a flexible controller may be indispensable to accomplish an improved performance. It is known that control system designers are not certain which controller (low/high order) should be selected to deliver better control to a specified process. Hence, it is appropriate to develop a new design method of IMC controller that 
works universally with an improved performance of common stable linear processes.

In this work, a novel more generalized IMC controller is proposed. The proposed controller design method has more flexibility when compared to conventional IMC controller design method. The proposed dynamic part of the controller and its parameters may be designed independently of the process model. The users have can choose between a low and a high-order controller. Loworder controllers such as PI/PID controllers are employed for industrial applications due to their simple structure, minimal parameter tuning and the possibility to achieve satisfactory performance. Hence, in this work, the controller structure is chosen as a simple first/second order type. Suppose, if the user has no restriction on the order of the controller, he/she can select it based on the process and requirements from a closedloop model. The paper is organized as follows: Section 2 explains the developed IMC controller and its design mainly based on extensively utilized process models with first-order time delay, with some evaluation of achievable performance. Simulation examples are presented in section 3 . Illustrative examples used show the applicability of the developed methodology for the high-order process are presented in section 4 . The derived conclusions are drawn in section 5 .

\section{Proposed IMC Controller Design}

The IMC scheme is represented in Figure 1; where $G_{P}(s)$ is the stable process transfer function which has to be controlled, $G_{m}(s)$ is the process transfer function model, $G_{P}(s)$ and $C(s)$ are the IMC controller transfer functions. In Figure $1 r, y$, and $d$ are the reference, process output, and input disturbance respectively.

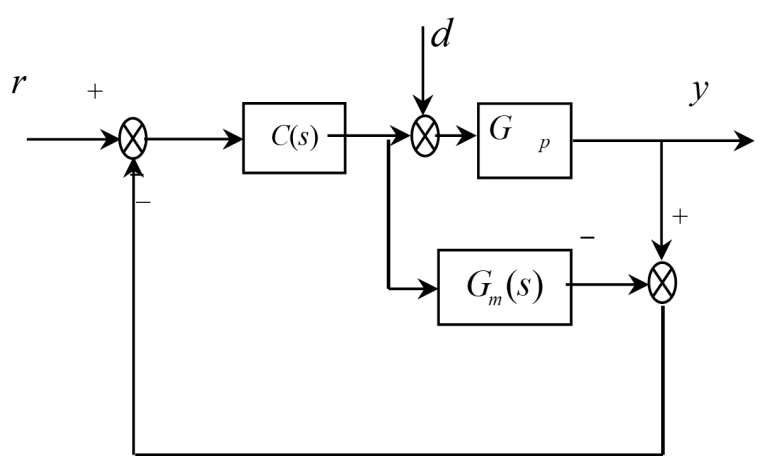

Figure 1. IMC Scheme (Morari \& Zafiriou, 1989)
The controller of IMC scheme is quantified as

$$
C(s)=G_{m-}(s)^{-1} f(s)
$$

where $G_{m-}(s)$ contains the minimum phase elements and $f(s)$ is a filter transfer function. Where there is no model plant mismatch, the open-loop IMC system between the set point and the process output is

$\frac{y(s)}{r(s)}=G_{m+}(s) f(s)$

where $G_{m+}(s)$ contains the non-minimum phase (NMP) elements of $G_{m}(s)$. The transfer function of controller filter $f(s)$ is usually chosen as

$$
f(s)=\frac{1}{(\lambda s+1)^{n}}
$$

The usually selected filter has the advantage of tuning only a single parameter; on the other hand, it limits the type of performance. It can be noticed that other choice of filter structure is not much focused.

\subsection{Proposed IMC Controller}

Let us assume that the minimum phase part $G_{m-}(s)$ of the model $G_{m}(s)$ can be factored into its gain and dynamic parts as shown below:

$G_{m-}(s)=k_{m} \bar{G}_{m-}(s)$

By substituting Equation (5) in Equation (2), one obtains

$C(s)=k_{m}{ }^{-1} \bar{G}_{m-}(s)^{-1} f(s)$

The IMC controller given in Equation 6 can be viewed in two ways: one as a gain element $\left(k_{m}^{-1}\right)$ and another one as a dynamic element $\left(\bar{G}_{m-}(s)^{-1} f(s)\right)$. The gain element cancellation between the controller and the process makes the forward loop a unity that ensures the set point tracking. The dynamic cancellation between the controller and the process achieves the specified behavior that is defined by a filter transfer function.

For the perfect model case, the IMC controller action can be viewed as follows: The controller gain element is the inverse of the process gain that computes process steady state input for the specified set point. This calculated input is passed through a dynamic compensator $\left(\bar{G}_{m-}(s)^{-1} f(s)\right)$, that

https://www.sic.ici.ro 
modifies the process input to improve the process response. The dynamic part determines the output transient response by shaping the process input. The conventional IMC achieves a particular type of performance due to the constraints such as model dependent and fixed filter structure. To achieve a flexible performance the controller structure should be flexible and also should have a generalized structure. Based on the views from above a generalized IMC controller $\left(C_{p}(s)\right)$ is proposed as:

$$
C_{p}(s)=k_{m}^{-1} D(s)
$$

where $D(s)$ is an $\mathrm{n}^{\text {th }}$-order dynamic compensator with unity gain.

The above-proposed modification in controller dynamic part develops a rationalized IMC controller. Further, the developed IMC controller gain part is the inverse of the process gain. In practice, the steady-state gain information of a process can be easily obtained.

By comparing the proposed (Equation 7) with the conventional IMC controller (Equation 2) the former has higher flexibility. The designer has the independence to design the dynamic element in order to meet the desired output response. The standard IMC controller can also be obtained from the proposed IMC controller, and it becomes one subset of the proposed IMC controller. The conventional IMC controller can be obtained from the proposed IMC controller by selecting the compensator as follows:

$$
D(s)=\bar{G}_{m-}(s)^{-1} f(s)
$$

Where there is no model plant mismatch, the transfer function of IMC system in between the setpoint and the process output for the proposed IMC controller is the following:

$$
\frac{y(s)}{r(s)}=G_{m+}(s) \bar{G}_{m-}(s) D(s)
$$

In general, $D(s)$ can be defined as follows:

$$
D(s)=\frac{b_{m} s^{m}+b_{m-1} s^{m-1}+\ldots \ldots+b_{1} s+1}{a_{n} s^{n}+a_{n-1} s^{n-1}+\ldots \ldots .+a_{1} s+1}
$$

where $n$ and $m$ are the integers. The designer has the option to choose the compensator order between a low to a high-order controller. As the compensator order increases then the number of controller tuning parameters will increase. The conventional IMC controller has the advantage of tuning only a single parameter irrespective of the process order. To limit the tuning parameters the compensator order may be selected as firstorder or second-order type irrespective of the process order.

\subsection{Design of Proposed IMC Controller}

A first-order process is generally modeled as

$G_{m}(s)=\frac{k_{m}}{\tau_{m} s+1} e^{-\theta s}$

where $k_{m}$ is the process gain, $\tau_{m}$ is the time constant and $\theta$ is the process time delay.

The present work proposes a new IMC design methodology. The proposed design idea is very simple: IMC controller dynamic part is a simple first-order compensator, and it is specified as:

$D(s)=\frac{b_{1} s+1}{a_{1} s+1}$

When there is no model plant mismatch, the transfer function of IMC system between the set point and the process output for the proposed IMC controller is the following:

$$
\frac{y(s)}{r(s)}=\frac{b_{1} s+1}{\left(a_{1} s+1\right)\left(\tau_{m} s+1\right)} e^{-\theta s}
$$

In the conventional IMC controller, the parameter ' $b_{1}$ ' is selected as the time constant of the process used to cancel the process dynamics. However, this cancelation yields a sluggish disturbance rejection performance. Hence, in this work, the compensator numerator parameter $\left(b_{1}\right)$ is considered a tuning parameter. The designer can alter the output response by tuning this parameter.

It is well-known that the process NMP elements such as dead-time and Right Half Plane (RHP) elements limit the closed loop performance improvement. The compensator denominator parameter $\left(a_{1}\right)$ can be selected with respect to NMP elements of process. In the most part of the process, the main limitation is the process deadtime. For process having dead-time the parameter $\left(a_{1}\right)$ is selected as process dead-time i.e.

$a_{1}=\theta$

where $\theta$ is the process dead-time. This choice may give a reasonable output response with right 
robustness margins and moderate input usage. Similar to traditional IMC controller, there is a single variable parameter $\left(b_{1}\right)$ in developed IMC controller that can be monotonically tuned to meet the compromise between achievable performance and the stability of a closed-loop system.

\subsection{Design of Proposed IMC Controller for Load Disturbance Rejection}

In the process control context load disturbance rejection is the most important issue. In this work, an IMC controller is framed to shape the disturbance response. The desired disturbance rejection characteristics can be specified regarding a transfer function. Therefore, for a controller designed to achieve the load disturbance rejection it is necessary to specify the transfer function of IMC system between the setpoint and the process output as

$\frac{y(s)}{r(s)}=\frac{b_{1} s+1}{\left(a_{1} s+1\right)^{2}} e^{-\theta s}$

To achieve the above response, the IMC controller dynamic part is computed as follows by using Equation (9)

$$
D(s)=\frac{\left(b_{1} s+1\right)\left(\tau_{m} s+1\right)}{\left(a_{1} s+1\right)^{2}}
$$

As stated earlier the parameter $\left(a_{1}\right)$ is selected as process dead-time. By tuning the parameter $\left(b_{1}\right)$, the response of load disturbance can be modified. The proposed design method holds the unique future of single parameter tuning of conventional IMC controller. A simple IMC controller design method is developed to increase the performance of disturbance rejection for slow processes with time delay with respect to broadly utilized first-order process models with time delay.

The appropriate selection of tuning parameter $b_{1}$ can achieve either the desired performance/ robustness. The optimal single tuning parameter can be designed by solving an optimization problem (Muniraj et al., 2017; Wenjie et al., 2020).

\section{Simulation Results}

The servo and regulatory performance of the development of the design methodology of
IMC controller is evaluated using simulation examples. The performance has been compared to the one of the conventional IMC controller and to the one of the feedback control scheme with PI/PID controller. Among several PID tuning relations, the well-known PID tuning rules of SIMC-PID (Skogestad, 2003) are considered in this comparative analysis. In all the simulation examples, in reference and input disturbance, a unit step change is induced to evaluate the performance.

\subsection{Robustness and Performance Metrics}

The standard IMC system shown in Figure 1 is redrawn into an equivalent single loop system as shown in Figure 2, if the single loop controller $G_{c}(s)$ is related to the IMC controller via (QingGuo et al., 2001)

$G_{c}(s)=\frac{C(s)}{1-C(s) G_{m}(s)}$

The loop transfer function of the feedback system can be expressed as:

$L(s)=G_{c}(s) G_{p}(s)$

The sensitivity functions that relate the output to the input signals are called indices.

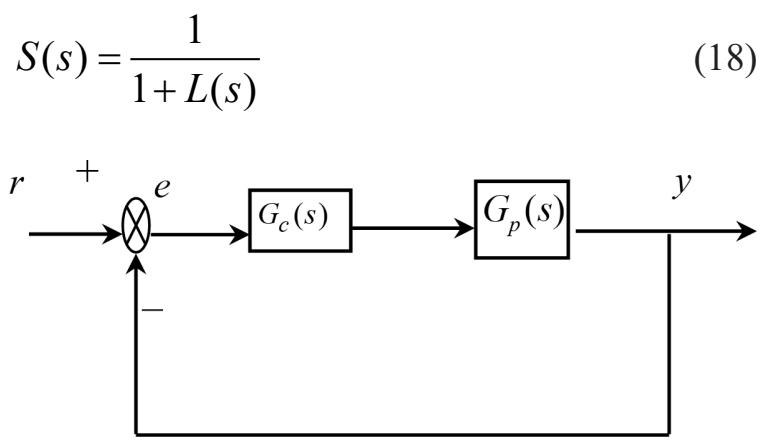

Figure 2. Single loop feedback Control Scheme

$S(s)$ are the sensitivity and the complementary sensitivity functions respectively. The sensitivity function describes the disturbance rejection properties while the complementary function provides a measure of set-point tracking performance. The sensitivity function fully depends on the loop transfer function and it can be visualized graphically in the Nyquist plot of the loop transfer function. The Nyquist plot is shown 
in Figure 3. The complex number $1+L(j \omega)$ can be represented as the vector from the point -1 to the point $L(j \omega)$ on the Nyquist curve (Astrom \& Hagglund, 2006). The maximum sensitivity is presented by,

$$
M s=\max _{\omega}\left|\frac{1}{1+G_{c}(j \omega) G_{p}(j \omega)}\right|
$$

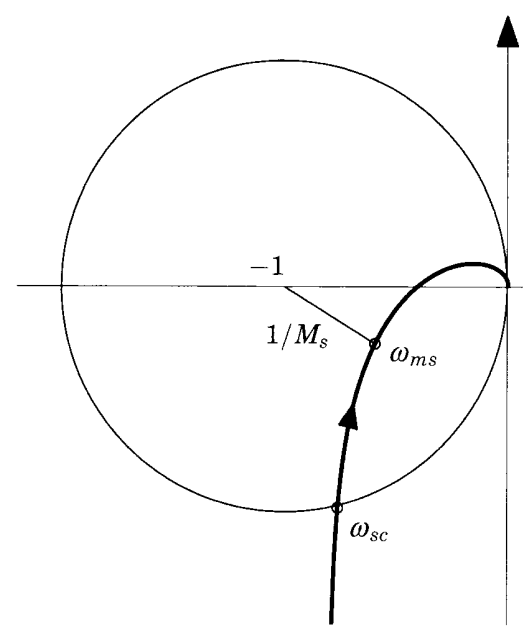

Figure 3. Nyquist curve of loop transfer function illustrating graphical interpretation of maximum sensitivity (Astrom \& Hagglund, 2006)

Notice that $|1+\mathrm{L}(\mathrm{j} \omega)|$ is the distance from a point on the Nyquist curve of the loop transfer function to the point -1 . The shortest distance from the Nyquist curve of the loop transfer function to the critical point -1 is thus $M s$. Maximum sensitivity $M s$ is a closed-loop system conventional measure of robustness. The characteristic values of $M s$ should be within the range 1.2-2.0. The range of $M s$ over 1.2-2.0 corresponds to gain margin of 6.0-2.0 and phase margin of 49.2-29.0 (Skogestad, 2003).

The commonly referred function and input usage measures such as Integral Absolute Error (IAE) and the Total variation (TV) of a manipulated input have been considered to calculate controller function. The IAE is given as

$$
I A E=\int_{0}^{\infty}|r(t)-y(t)| d t
$$

The TV of the controller output $u$ is evaluated as

$$
T V=\sum_{k=1}^{\infty}|u(k+1)-u(k)|
$$

\section{Example 1}

By considering the first-order plus dead-time process $G_{m}(s)=\frac{1}{s+1} e^{-0.5 s}$, the proposed IMC controller is designed for load disturbance rejection using (15). The performance (IAE, TV) and robustness $(M S)$ metrics of the proposed and conventional IMC controller and SIMC-PI controller are computed for various values of the tuning parameters $b_{1}$ and $\lambda$ respectively. The computed values are shown in Figures 4-6.
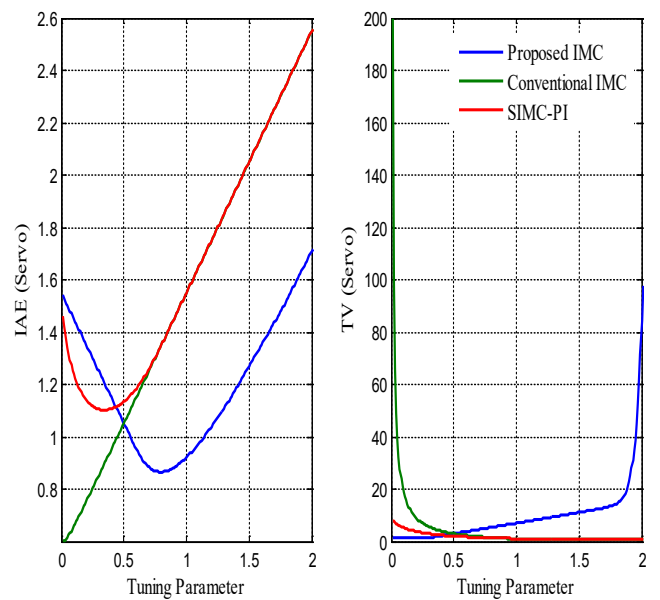

Figure 4. Servo performance analysis for first-order plus dead-time process
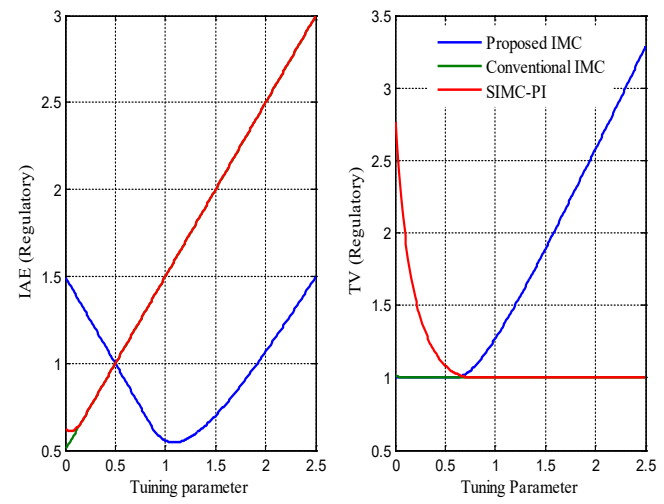

Figure 5. Regulatory performance analysis for firstorder plus dead-time process

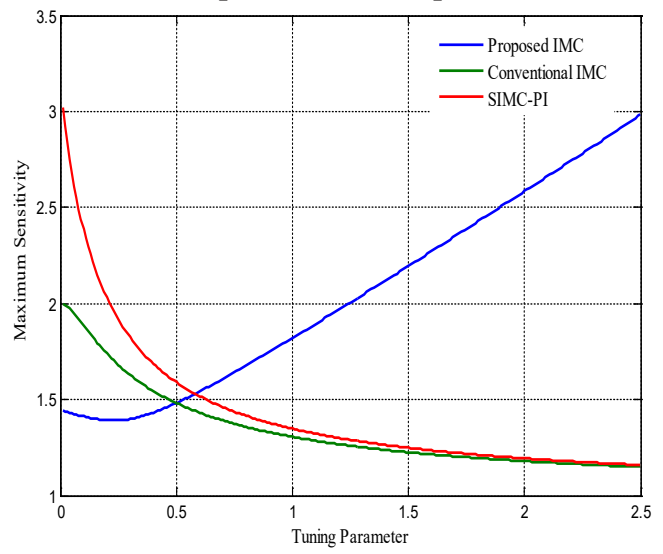

Figure 6. Robustness analysis for first-order plus dead-time process 
The IAE values of the conventional IMC controller of servo and regulatory responses vary linearly with the tuning parameter $\lambda$ i.e. the IAE increases as $\lambda$ increases. But for the proposed IMC controller the relationship is nonlinear with the tuning parameter $b_{1}$. The servo performance TV of conventional IMC controller is high for lower values of $\lambda$ and becomes constant for higher values.

Moreover, the regulatory performance TV is constant irrespective of $\lambda$. The servo performance TV of the proposed IMC controller is constant for the minimum values of $b_{1}$ and increases for the maximum values of $b_{1}$. Similarly, the regulatory performance TV is initially constant and linearly increases for maximum values of $b_{1}$. The performance of SIMC-PI controller is almost similar to conventional IMC controller.

The $M s$ values of the traditional IMC controller decrease as $\lambda$ increases, i.e., the robustness increases with the increasing values of $\lambda$. But the robustness of proposed IMC controller increases for decreasing value of $b_{1}$. Hence lower values of $b_{1}$ should be selected to give enough robustness.

The developed IMC controller has minimum IAE value for a certain value of $b_{1}$. The user can select that $b_{1}$ value as the controller parameter. The proposed IMC controller parameter $\left(b_{s 1}=0.81\right)$ is chosen for servo performance and $b_{r 1}=1.09$ for regulatory performance. A two-degree-offreedom IMC controller is implemented to achieve better servo and regulatory performance. For comparison, the parameters of the conventional IMC and SIMC-PI controllers are chosen to yield the same robustness of the proposed IMC controller as seen in Figure 6. The corresponding $T V$ values are obtained as illustrated in Figures 3 and 4 . The obtained performance and robustness values are presented in Table 1. Servo-regulatory response and controller output of all the control techniques are exhibited in Figure 7.

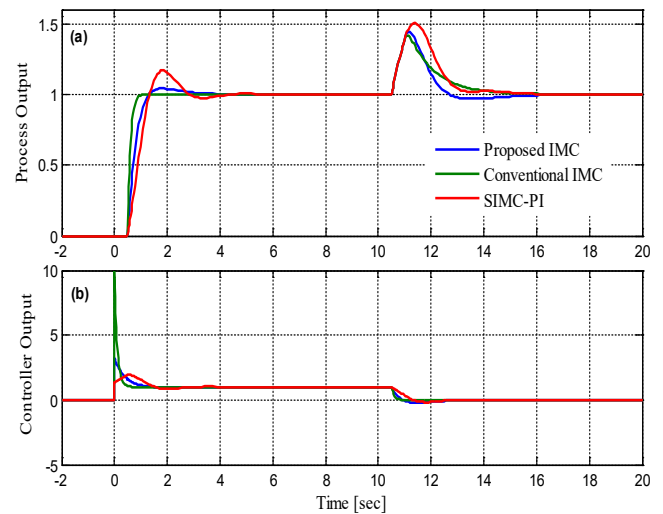

Figure 7. (a) Servo and Regulatory Responses; (b) Controller Outputs for the FOPDT Process Model
Table 1. Performance Comparison for FOPDT Process

\begin{tabular}{|c|c|c|c|c|c|c|}
\hline \multirow{2}{*}{ Method } & \multirow{2}{*}{$\begin{array}{c}\text { Controller } \\
\text { parameter }\end{array}$} & $\boldsymbol{M S}$ & \multicolumn{2}{|c|}{ Setpoint } & \multicolumn{2}{|c|}{ Regulatory } \\
\cline { 4 - 7 } & $\boldsymbol{I A E}$ & $\boldsymbol{T V}$ & $\boldsymbol{I A E}$ & $\boldsymbol{T V}$ \\
\hline $\begin{array}{c}\text { Proposed } \\
\text { IMC } \\
\text { Controller }\end{array}$ & $\begin{array}{l}b_{s 1}=0.81 \\
b_{r 1}=1.09 \\
a_{1}=0.5\end{array}$ & 1.89 & 0.81 & 5.49 & 0.54 & 1.37 \\
\hline $\begin{array}{c}\text { Conventional } \\
\text { IMC } \\
\text { Controller }\end{array}$ & $\lambda=0.1$ & 1.89 & 0.60 & 19.0 & 0.60 & 1.02 \\
\hline $\begin{array}{c}\text { SIMC-PI } \\
\boldsymbol{k}_{c}=1.3 \\
\tau_{i}=1 \\
\lambda=0.27\end{array}$ & 1.89 & 1.06 & 3.32 & 0.76 & 1.41 \\
\hline
\end{tabular}

The developed IMC controller exhibits an increased regulatory performance with comparable controller output performance (TV) when differentiated with conventional IMC and SIMC-PI controllers. The servo performance of the proposed IMC controller is better than the one of the SIMC-PI controller. Conventional IMC controller gives good servo performance at the cost of higher input usage. By assuming an error of $25 \%$ in the process-parameter estimation, the time constant process decreases with $25 \%$ and the time delay increases with $25 \%$. The corresponding regulatory response is presented in Figure 8, indicating that developed IMC controller outperforms the uncertainties in process.

The IMC controller that designed with the same robustness as the one of the proposed IMC controller shows unstable performance for a perturbed system. But, the proposed IMC controller has an improved regulatory performance and also an improved robustness when compared to the conventional controller. The IMC controller shows good servo performance but poor robustness when compared to proposed IMC and SIMC-PI controllers. The SIMC-PI controller that is derived from conventional IMC controller shows a robust function in the presence of uncertainties.

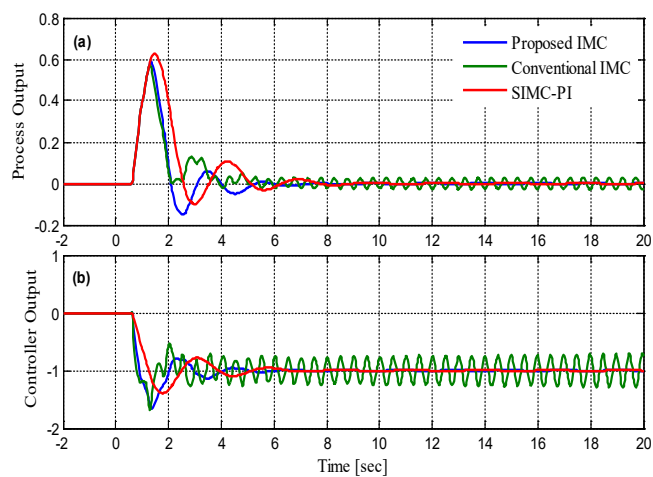

Figure 8. (a) Perturbed System Servo and Regulatory Responses; (b) Controller Outputs for FOPDT Process Model 


\section{Example 2}

By considering a lag-dominated first-order plus dead-time process $G_{m}(s)=\frac{1}{100 s+1} e^{-30 s}$, the developed IMC controller is modeled for load disturbance rejection using (15). The servo, regulatory performance (IAE, TV) and robustness measure $(M S)$ of the proposed and conventional IMC and SIMC-PI controllers are computed for various values of tuning parameters $\lambda$ and $b_{1}$ respectively. The computed values are shown in Figures 9-11.
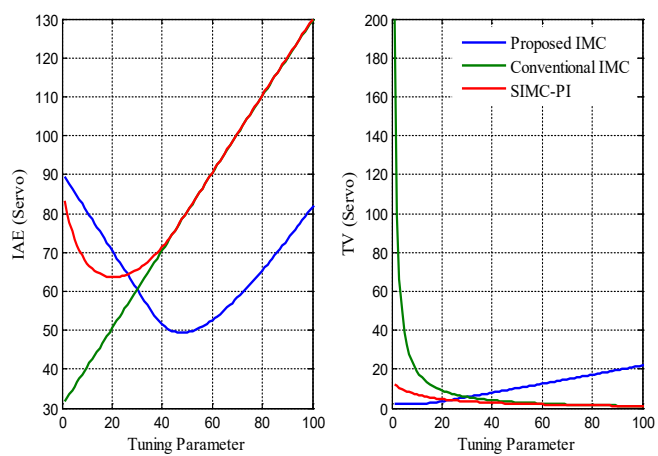

Figure 9. Servo performance analysis for lagdominated first-order plus dead-time process
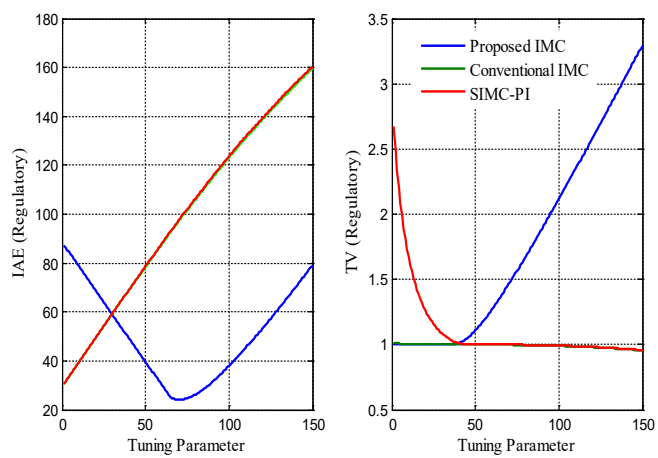

Figure 10. Regulatory performance analysis for lagdominated first-order plus dead-time process

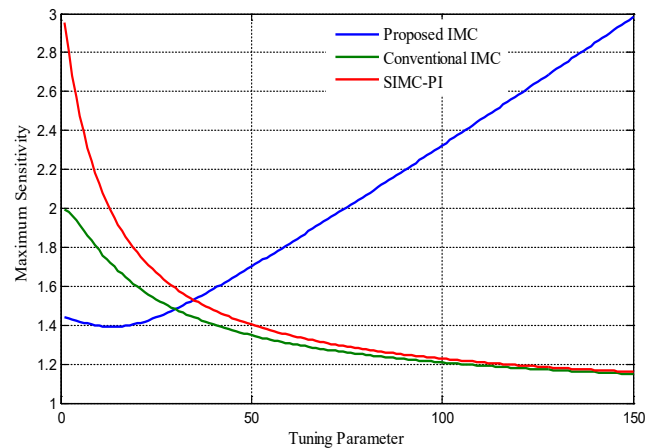

Figure 11. Robustness analysis for lag-dominated first-order plus dead-time process

The proposed IMC controller parameter is chosen for minimum $I A E$ servo and regulatory performance. The parameter of the conventional
IMC and SIMC-PI controllers is modeled to give same robustness as the one of the proposed IMC controller. Further, computed performance and robustness measures are exhibited in Table 2.

Table 2. Performance Comparison for lag-dominated FOPDT Process

\begin{tabular}{|c|c|c|c|c|c|c|}
\hline \multirow{2}{*}{ Method } & \multirow{2}{*}{$\begin{array}{c}\text { Controller } \\
\text { parameter }\end{array}$} & \multirow{2}{*}{$\boldsymbol{M S}$} & \multicolumn{2}{|c|}{ Setpoint } & \multicolumn{2}{|c|}{ Regulatory } \\
\cline { 4 - 7 } & & $\boldsymbol{I A E}$ & $\boldsymbol{T V}$ & $\boldsymbol{I A E}$ & $\boldsymbol{T V}$ \\
\hline $\begin{array}{c}\text { Proposed } \\
\text { IMC } \\
\text { Controller }\end{array}$ & $\begin{array}{c}b_{s 1}=71 \\
b_{r 1}=48 \\
a_{1}=30\end{array}$ & 1.96 & 48.80 & 9.71 & 24.32 & 1.46 \\
\hline $\begin{array}{c}\text { Conventional } \\
\text { IMC } \\
\text { Controller }\end{array}$ & $\lambda=3$ & 1.96 & 33.0 & 65.67 & 32.95 & 1.07 \\
\hline SIMC-PI & $\begin{array}{l}k_{c}=2.3 \\
\tau_{i}=100 \\
\lambda=14\end{array}$ & 1.96 & 64.56 & 5.87 & 43.42 & 1.47 \\
\hline
\end{tabular}

The control techniques, servo-regulatory response and controller output are shown in Figure 12. Performance metrics (IAE and TV) and responses indicate that the proposed IMC controller shows an improved regulatory performance.

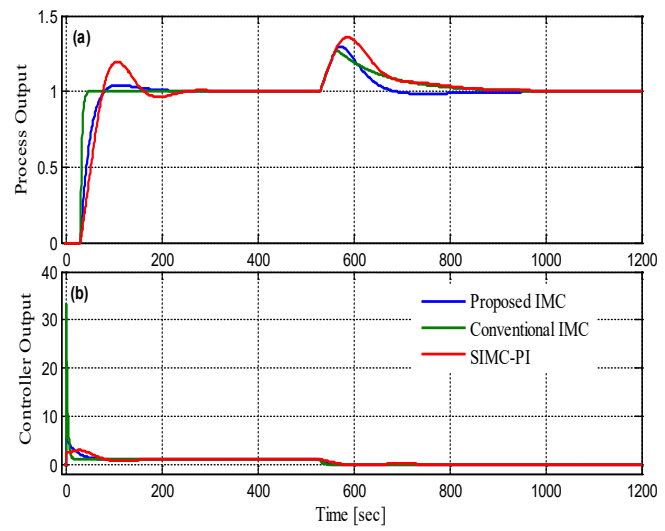

Figure 12. (a) Servo and Regulatory Responses; (b) Controller Outputs for the lag-dominated FOPDT Process Model

By assuming an error of $25 \%$ in the processparameter estimation, the time constant process decreases with $25 \%$ and the time delay increases with $25 \%$. The output responses are exhibited in Figure 13, indicating that the developed IMC controller outperforms uncertainties in process. Similar to the previous example the proposed IMC controller shows a robust performance when compared to conventional IMC controller. The conventional IMC controller designed for the same robustness as the one of proposed IMC controller shows unstable response. 

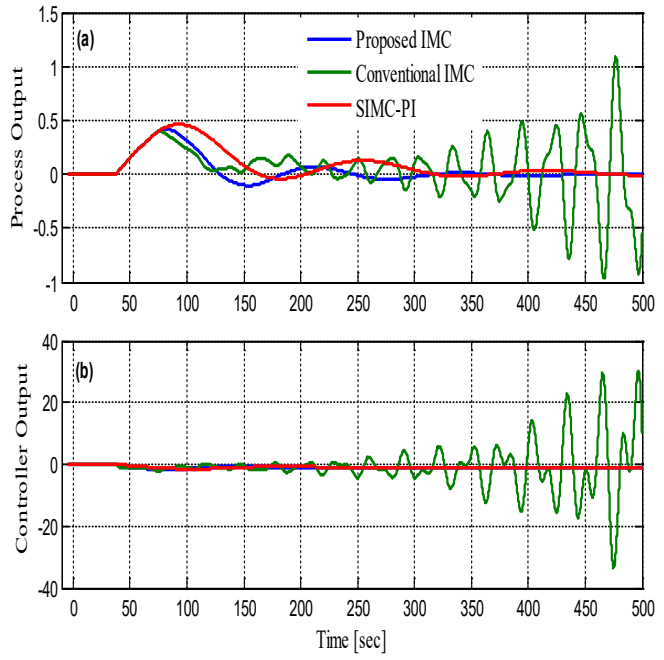

Figure 13. (a) Perturbed System Regulatory Responses; (b) Controller Outputs for the lagdominated FOPDT Process Model

\section{Example 3}

By considering the following secondorder plus dead-time process model $G_{m}(s)=\frac{1}{(20 s+1)(2 s+1)} e^{-s}$, the proposed IMC controller is designed for load disturbance rejection using (15). The servo, regulatory performance $(I A E, T V)$ and robustness measure $(M s)$ of the proposed and conventional IMC and SIMC-PI controllers are computed for various values of the tuning parameters $\lambda$ and $b_{1}$ respectively. The computed values are shown in Figures 14-16. The proposed IMC controller parameter is chosen for minimum IAE servo and regulatory performance. The conventional IMC and SIMC-PI controller parameter is designed to give the same robustness as the one of proposed IMC controller.
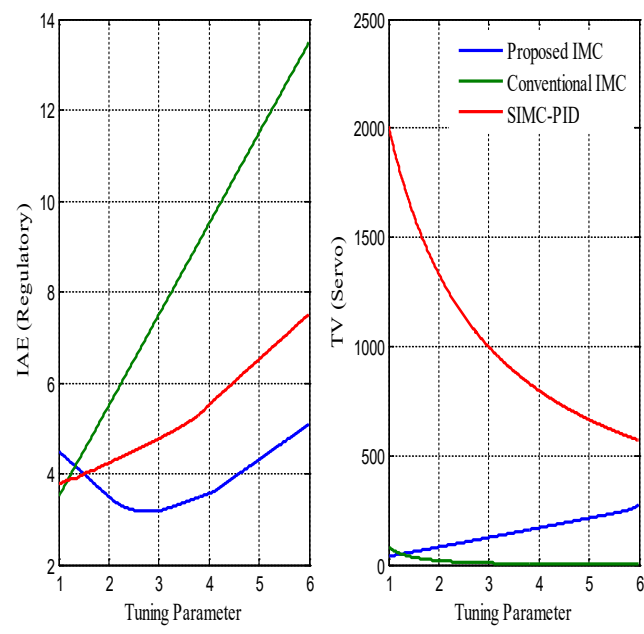

Figure 14. Servo performance analysis for lagdominated second-order plus dead-time process
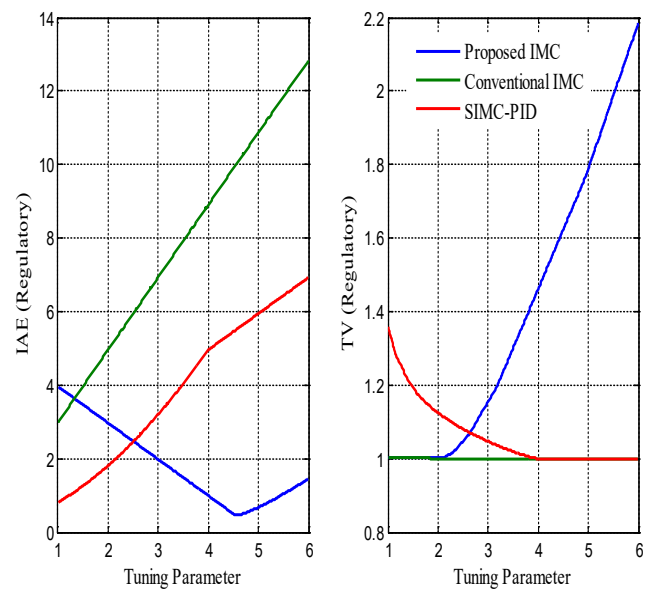

Figure 15. Regulatory performance analysis for lagdominated second-order plus dead-time process

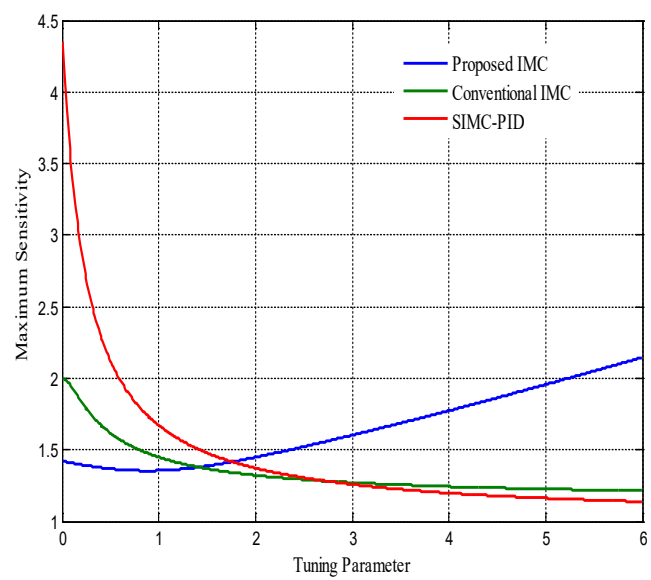

Figure 16. Robustness analysis for lag-dominated second-order plus dead-time process

The computed performance measures are presented in Table 3. The servo-regulatory response and controller output of control schemes are illustrated in Figure 17-18 respectively. The performance metrics (IAE and $T V$ ) and responses indicate that the developed IMC controller shows an increased regulatory performance in comparison with the conventional IMC and SIMC-PID controllers.

Table 3. Performance Comparison for lag-dominated SOPDT Process

\begin{tabular}{|c|c|c|c|c|c|c|}
\hline \multirow{2}{*}{ Method } & \multirow{2}{*}{$\begin{array}{c}\text { Controller } \\
\text { parameter }\end{array}$} & \multirow{2}{*}{$\boldsymbol{M S}$} & \multicolumn{2}{|c|}{ Setpoint } & \multicolumn{2}{|c|}{ Regulatory } \\
\cline { 4 - 7 } & & $\boldsymbol{I A E}$ & $\boldsymbol{T V}$ & $\boldsymbol{I A E}$ & $\boldsymbol{T V}$ \\
\hline $\begin{array}{c}\text { Proposed } \\
\text { IMC } \\
\text { Controller }\end{array}$ & $\begin{array}{l}b_{s 1}=3.0 \\
b_{r 1}=4.6 \\
a_{1}=1\end{array}$ & 1.88 & 2.69 & 124.92 & 0.46 & 1.68 \\
\hline $\begin{array}{c}\text { Conventional } \\
\text { IMC } \\
\text { Controller }\end{array}$ & $\lambda=0.16$ & 1.88 & 1.32 & 3475 & 1.29 & 2.31 \\
\hline SIMC-PI & $\begin{array}{l}k_{c}=11.77 \\
\tau_{i}=6.8 \\
\lambda=0.7\end{array}$ & 1.88 & 3.91 & 29.51 & 0.58 & 1.57 \\
\hline
\end{tabular}




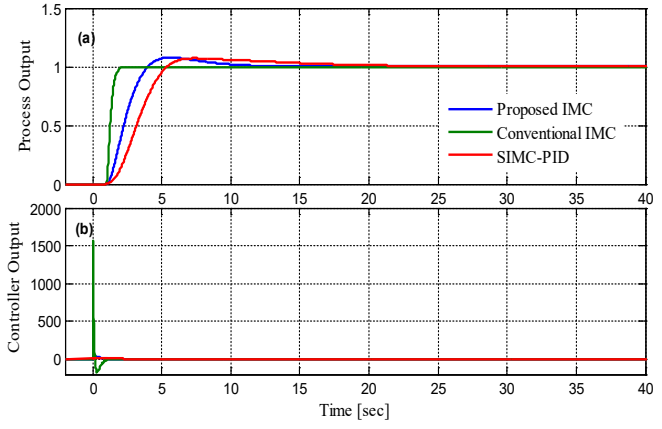

Figure 17. (a) Servo Responses; (b) Controller Outputs for the Lag-dominated SOPDT Process Model

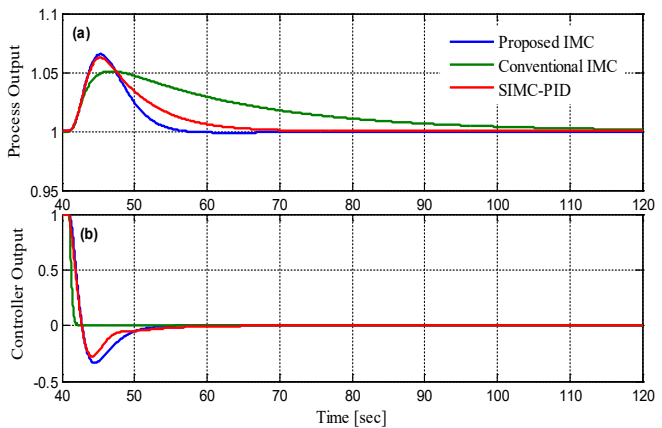

Figure 18. (a) Regulatory Responses; (b) Controller Outputs for the Lag-dominated SOPDT Process Model

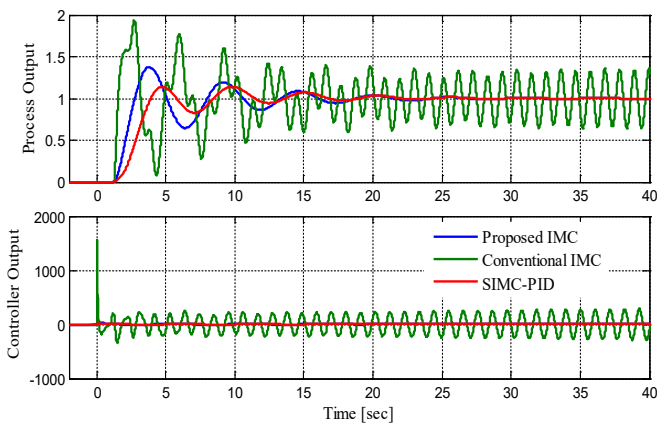

Figure 19. (a) Perturbed System Servo Responses; (b) Controller Outputs for the SOPDT Process Model

By assuming an error of $25 \%$ in the processparameter estimation, the time constant process decreases with $25 \%$ and the time delay increases with $25 \%$. The corresponding output responses are presented in Figure 19, indicating that the developed IMC controller outperforms uncertainties in process. It can be noticed that the conventional IMC controller shows unstable response.

\section{Extension to High-order Process}

The servo and regulatory function of the developed design methodology of IMC controller is are evaluated using simulation examples for high-order processes. The performance has been compared to the one of the conventional IMC controller and of a feedback control scheme with PI/PID controller.

\section{Example 4}

By considering the following fourth-order dead-time process $G_{m}(s)=\frac{1}{(s+1)(0.5 s+1)(0.25 s+1)} e^{-3 s}$, the $(0.125 s+1)$

proposed IMC controller is designed using (11). The developed and conventional IMC controllers are modeled to in order to achieve a robustness similar to the one of the SIMC-PID controller with a maximum sensitivity ( $M s)$ of 1.59 . The controller parameters are shown in Table 4.

Table 4. Performance comparison for fourth-order dead-time process

\begin{tabular}{|c|c|c|c|c|c|c|}
\hline \multirow{2}{*}{ Method } & \multirow{2}{*}{$\begin{array}{l}\text { Controller } \\
\text { parameter }\end{array}$} & $\boldsymbol{M S}$ & \multicolumn{2}{|c|}{ Setpoint } & \multicolumn{2}{|c|}{ Regulatory } \\
\cline { 3 - 7 } & $\boldsymbol{I A E}$ & $\boldsymbol{T V}$ & $\boldsymbol{I A E}$ & $\boldsymbol{T V}$ \\
\hline $\begin{array}{c}\text { Proposed } \\
\text { IMC } \\
\text { Controller }\end{array}$ & $\begin{array}{l}b_{1}=1.75 \\
a_{1}=3\end{array}$ & 1.59 & 6.12 & 1.0 & 6.12 & 1.0 \\
\hline $\begin{array}{c}\text { Conventional } \\
\text { IMC } \\
\text { Controller }\end{array}$ & $\lambda=1.9$ & 1.59 & 10.6 & 1.0 & 10.6 & 1.0 \\
\hline SIMC-PI & $\begin{array}{l}k_{c}=0.153 \\
\tau_{i}=1.0 \\
\tau_{d}=0.625 \\
\lambda=3.25\end{array}$ & 1.59 & 7.67 & 1.2 & 7.02 & 1.12 \\
\hline
\end{tabular}

The servo-regulatory performance of the control techniques is presented in Figure 20.
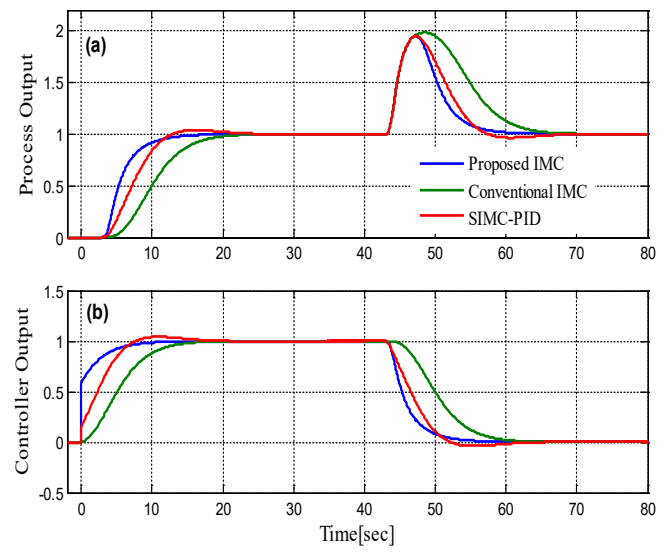

Figure 20. (a) Servo-regulatory Responses; (b) Controller Outputs for the Fourth-order dead-time process 
The performance measures (IAE and $T V$ ) indicate that the developed IMC controller exhibits superior servo-regulatory performance when compared to the conventional IMC controller. Also, the control schemes have similar control efforts. Further, the proposed IMC controller achieves an improved servo-regulatory performance with less control effort when compared to the SIMC-PID control scheme.

It can be noticed that the proposed IMC controller has a first-order type compensator whereas the compensator of the conventional IMC controller is a fourth-order type and it is also a function of the dynamic process model. From this simulation analysis, it can be inferred that a better process response can be achieved with proper tuning of a low-order compensator for the high-order deadtime processes.

\section{Example 5}

By considering the following high-order process with dead-time and RHP zero $G_{m}(s)=\frac{-s+1}{(s+1)^{5}} e^{-2 s}$, the proposed IMC controller is designed using (11). The developed and conventional IMC controllers are modeled in order to achieve a robustness similar to the one of the SIMC-PID controller with a maximum sensitivity ( $M s$ ) of 1.57 . The controller parameters and the calculation of the performance measures are reported in Table 5. The proposed IMC controller parameter $a_{1}$ is set at 4 . By considering the process dead-time and RHP element, the above value has been selected from the system open-loop response. The servoregulatory performance of the control techniques is presented in Figure 21.

Table 5. Performance Comparison for High-order dead-time and RHP zero process

\begin{tabular}{|c|c|c|c|c|c|c|}
\hline \multirow{2}{*}{ Method } & \multirow{2}{*}{$\begin{array}{l}\text { Controller } \\
\text { parameter }\end{array}$} & \multirow{2}{*}{$\boldsymbol{M S}$} & \multicolumn{2}{|c|}{ Setpoint } & \multicolumn{2}{|c|}{ Regulatory } \\
\cline { 4 - 7 } & & $\boldsymbol{I A E}$ & $\boldsymbol{T V}$ & $\boldsymbol{I A E}$ & $\boldsymbol{T} \boldsymbol{V}$ \\
\hline $\begin{array}{c}\text { Proposed } \\
\text { IMC } \\
\text { Controller }\end{array}$ & $\begin{array}{l}b_{1}=1.5 \\
a_{1}=4\end{array}$ & 1.57 & 10.5 & 1.0 & 10.6 & 1.04 \\
\hline $\begin{array}{c}\text { Conventional } \\
\text { IMC } \\
\text { Controller }\end{array}$ & $\lambda=3.1$ & 1.57 & 18.5 & 1.0 & 18.6 & 1.0 \\
\hline SIMC-PI & $\begin{array}{l}k_{c}=0.136 \\
\tau_{i}=1.5 \\
\tau_{d}=1.0 \\
\lambda=5.5\end{array}$ & 1.57 & 12.95 & 1.16 & 12.0 & 1.13 \\
\hline
\end{tabular}
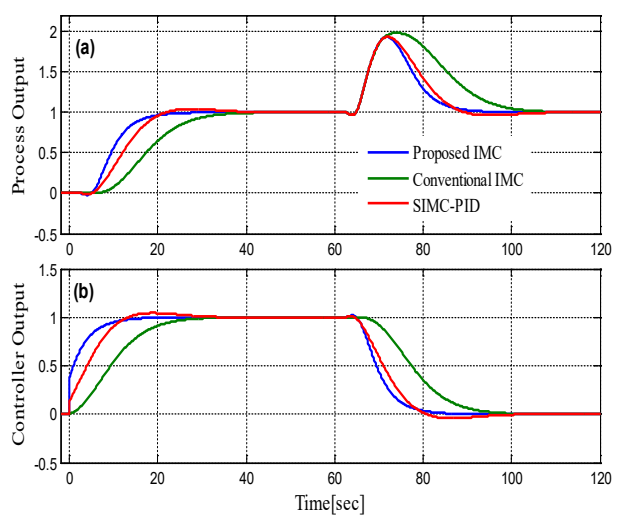

Figure 21. (a) Servo-regulatory Responses; (b) Controller Outputs for the High-order dead-time and RHP Zero process

Similar to the previous example the proposed IMC controller shows superior servo-regulatory performance with similar control efforts when compared to conventional IMC controller. Also, the developed IMC controller has an increased servo-regulatory function with less control effort when to the SIMC-PID control technique. From the simulation analysis, it can be inferred that a better process response is achieved with proper tuning of a low-order compensator for the highorder dead-time and RHP zero process.

The conventional IMC controller shows poor servo-regulatory performance when compared to SIMC-PID controller. Hence, there is a need for a modified or improved controller design for IMC scheme. The proposed modified IMC controller outperforms the conventional IMC and SIMCPID controllers when it is tuned for the same robustness specification.

\section{Conclusion}

A new controller design methodology for IMC scheme has been developed in this paper. The proposed controller design method of the IMC controller is flexible when compared to the traditional IMC controller. The proposed controller compensator order has been considered to be of the first/second-order type, and the design guidelines have been presented. Similar to the conventional IMC controller the proposed IMC controller has a single tuning parameter that can be tuned to achieve satisfactory performance. An extensive simulation analysis demonstrates that the proposed IMC controller offers an increased disturbance rejection response when compared 
to the conventional IMC controller and SIMCPI/PID feedback controller. Also, the proposed IMC controller shows robust performance when compared to the conventional IMC controller in the presence of plant model mismatches.
Moreover, the simulation results turn out that the proposed low-order IMC controller achieves an improved servo-regulatory performance when compared to the conventional IMC controller for high-order process.

\section{REFERENCES}

Alcantara, S., Pedret, C. \& Vilanova, R. (2010). On the model matching approach to PID design: analytical perspective for robust servo/regulator trade off tuning, Journal of Process Control, 20(5), 596-608.

Arun, R. P. \& Prakash, J. (2018). Design of Internal Model Control Dead-time Scheme for First Order Plus Dead-time Systems, Canadian Journal of Chemical Engineering, 96(12), 2553-2563.

Astrom, K. J. \& Hagglund, T. (2006). Advanced PID Control. ISA, Research Triangle Park, NC.

Canale, M., Fagiano, L., Ferrara, A. \& Vecchio, C. (2009). Comparing Internal Model Control and Sliding-Mode Approaches for Vehicle Yaw Control, IEEE Transactions on Intelligent Transport System, 10(1), 31-41.

Chau, P. C. (2002). Process Control: A First Course with MATLAB. Cambridge University Press, New York.

Jha, S. K., Yadav, A. K., Gaur, P., Gupta, J. R. P. \& Parthasarathy, H. (2014). Robust and Optimal Control Analysis of Sun Seeker System, Journal of Control Engineering and Applied Informatics, 16(1), 70-79.

Lennartson, B. \& Kristiannson, B. (2009). Evaluation and Tuning of Robust PID Controllers, IET Control Theory and Applications, 3(3), 294-302.

Li, S. \& Gu, H. (2012). Fuzzy Adaptive Internal Model Control Schemes for PMSM Speed Regulation System, IEEE Transactions on Industrial Informatics, 8(4), 767-79.

Liu, T. \& Gao, F. (2010). New insight into internal model control filter design for load disturbance rejection, IET Control Theory Applications, 4(3), 448-460.

Morari, M. \& Zafiriou, E. (1989). Robust Process Control. Prentice-Hall, New Jersey.

Rathinam, M., Maria Siluvairaj, W. I. \& Arun, Ramaveerapathiran, A. (2017). Tuning of Robust PID controller with Filter for SISO system using Evolutionary Algorithms, Studies in Informatics and Control, 26(3), 277-286. DOI: 10.24846/ v26i3y201703
Qing-Guo, W., Hang, C. C. \& Yang, X. P. (2001). Single -Loop Controller Design via IMC Principles, Automatica, 37(12), 2041-2048.

Rupp, D. \& Guzzella, L. (2010). Adaptive internal model control with application to fueling control, Control Engineering Practice, 18(8), 873-81.

Saxena, S. \& Hote, Y. V. (2013). Load Frequency Control in Power Systems via Internal Model Control Scheme and Model-Order Reduction, IEEE Transactions on Power System, 28(3), 2749-2757.

Seborg. D. E., Edgar, T. F. \& Mellichamp, D. A. (2004). Process Dynamics and Control. John Wiley \& Sons, New York.

Shamsuzzoha, M. \& Lee, M. (2007). IMC-PID controller design for improved disturbance rejection of time-delayed processes, Industrial \& Engineering Chemistry, 46(7), 2077-2091.

Skogestad, S. (2003). Simple Analytic Rules for Model Reduction and PID Controller Tuning, Journal of Process Control, 13(4), 291-309.

Van de Vegte, J. (1986). Feedback Control Systems, Sec. 1.8. Englewood Cliffs, Prentice Hall, Inc., New Jersey.

Wang, Q.-G. \& Zhang, Y. (2001). Robust identification of continuous systems with dead-time from step responses, Automatica, 37(3), 377-390.

Wenjie, Z., Weicong, Z., Tianyu, H., Lezhi, C., Jinsen, X. \& Tao, Y. (2020). An IMC-PID controller with Particle Swarm Optimization algorithm for MSBR core power control, Nuclear Engineering and Design, $360,1-7$.

Yadav, A. K. \& Gaur, P. (2013). Comparative analysis of modern control and AI-based control for maintaining constant ambient temperature, World Journal of Science, Technology and Sustainable Development, 10, 56-77.

Zitek, P. \& Halva, J. (2001). Anisochronic internal model control of time-delay systems, Control Engineering Practice, 9(5), 501-516. 